\title{
Anesthetics to Prevent Lung Injury in Cardiac Surgery (APLICS): a protocol for a randomized controlled trial
}

Brian O'Gara* D, Balachundhar Subramaniam, Shahzad Shaefi, Ariel Mueller, Valerie Banner-Goodspeed and Daniel Talmor

\begin{abstract}
Background: Patients undergoing cardiac surgery with cardiopulmonary bypass are at an increased risk of developing postoperative pulmonary complications, potentially leading to excess morbidity and mortality. It is likely that pulmonary ischemia-reperfusion (IR) injury during cardiopulmonary bypass is a major contributor to perioperative lung injury. Therefore, interventions that can minimize IR injury would be valuable in reducing the excess burden of this potentially preventable disease process. Volatile anesthetics including sevoflurane have been shown in both preclinical and human trials to effectively limit pulmonary inflammation in a number of settings including ischemia-reperfusion injury. However, this finding has not yet been demonstrated in the cardiac surgery population. The Anesthetics to Prevent Lung Injury in Cardiac Surgery (APLICS) trial is a randomized controlled trial (RCT) investigating whether sevoflurane anesthetic maintenance can modulate pulmonary inflammation occurring during cardiac surgery with cardiopulmonary bypass and whether this potential effect can translate to a reduction in postoperative pulmonary complications.

Methods: APLICS is a prospective RCT of adult cardiac surgical patients. Participants will be randomized to receive intraoperative anesthetic maintenance with either sevoflurane or propofol. Patients in both groups will be ventilated according to protocols intended to minimize the influences of ventilator-induced lung injury and hyperoxia. Bronchoalveolar lavage (BAL) and blood sampling will take place after anesthetic induction and $2-4 \mathrm{~h}$ after pulmonary reperfusion. The primary outcome is a difference between groups in the degree of post-bypass lung inflammation, defined by BAL concentrations of TNFa. Secondary outcomes will include differences in additional relevant BAL and systemic inflammatory markers and the incidence of postoperative pulmonary complications.
\end{abstract}

Discussion: APLICS investigates whether anesthetic choice can influence lung inflammation and pulmonary outcomes following cardiac surgery with cardiopulmonary bypass. A positive result from this trial would add to the growing body of evidence describing the lung protective properties of the volatile anesthetics and potentially reduce unnecessary morbidity for cardiac surgery patients.

Trial registration: ClinicalTrials.gov, NCT02918877. Registered on 29 September 2016.

Keywords: Inflammatory lung injury, Volatile anesthetics, Cardiac surgery, Postoperative pulmonary complications, TNFa

\footnotetext{
*Correspondence: bpogara@bidmc.harvard.edu

Department of Anesthesia, Critical Care and Pain Medicine, Beth Israel

Deaconess Medical Center, Harvard Medical School, 330 Brookline Ave,

Boston, MA 02215, USA
}

(c) The Author(s). 2019 Open Access This article is distributed under the terms of the Creative Commons Attribution 4.0 International License (http://creativecommons.org/licenses/by/4.0/) which permits unrestricted use, distribution, and reproduction in any medium, provided you give appropriate credit to the original author(s) and the source, provide a link to the Creative Commons license, and indicate if changes were made. The Creative Commons Public Domain Dedication waiver (http://creativecommons.org/publicdomain/zero/1.0/) applies to the data made available in this article, unless otherwise stated. 


\section{Background}

Patients undergoing cardiac surgery with cardiopulmonary bypass (CPB) are at an increased risk for postoperative pulmonary complications (PPCs), with recent estimates of the incidence as high as $50 \%[1,2]$. Overt respiratory failure and acute respiratory distress syndrome (ARDS) can occur in as many as $10 \%$ of patients, leading to mortality rates up to 40 times higher than patients without these conditions [3]. Pulmonary ischemia-reperfusion (IR) injury during CPB has been identified as a major contributor to perioperative lung injury, but to date strategies to mitigate such injury have failed to demonstrate a consistent benefit $[4,5]$. Volatile anesthetics have been shown to protect the lung from various etiologies of inflammatory lung injury in both preclinical models and in humans [6-9]. Proposed mechanisms for this observed effect include a reduction in the release of inflammatory mediators from pulmonary neutrophils and macrophages and preservation of alveolar endothelial integrity [10]. Although the volatile anesthetic sevoflurane has been shown to prevent myocardial injury from IR after cardiac surgery, there has been a lack of data showing whether its use in this population can result in a similar level of protection from IR lung injury [11]. Given the excess morbidity and mortality associated with PPCs in this population, investigation into whether the use of volatile anesthetics such as sevoflurane can reduce lung injury after CPB could impact the outcomes of thousands of cardiac surgical patients a year [12].

\section{Methods and design \\ Study design}

The Anesthetics to Prevent Lung Injury in Cardiac Surgery (APLICS) trial is a randomized, controlled, singlecenter clinical trial of adult cardiac surgical patients undergoing $\mathrm{CPB}$. Individuals are randomized to receive intraoperative anesthetic maintenance with either sevoflurane or propofol. Lung inflammation will be evaluated by analyzing differences in the pre- and post-bypass pulmonary tumor necrosis factor alpha (TNF $\alpha$ ) between groups, obtained via a bronchoalveolar lavage (BAL).
Secondary outcomes include differences in other key pulmonary and systemic inflammatory biomarkers, as well as the incidence of PPCs. A study schema is provided in Fig. 1 and Additional file 2.

\section{Setting}

This study is being conducted at Beth Israel Deaconess Medical Center (BIDMC) in Boston. BIDMC is a 700bed tertiary-care hospital academically affiliated with Harvard Medical School. More than 900 open-heart procedures with $\mathrm{CPB}$ are performed at BIDMC each year.

\section{Study registration}

Institutional Review Board (IRB) approval was obtained from the Committee on Clinical Investigations at BIDMC (IRB Protocol no. 2016P000306). APLICS was registered on clinicaltrials.gov with the identifier NCT02918877. Upon completion of the trial, results will be reported according to the Consolidated Standards of Reporting Trials (CONSORT) guidelines and the Standard Protocols Items: Recommendations for Interventional Trials (Fig. 2 and Additional file 1). The trial is active and ongoing; any amendments made to the protocol are reported to and approved by the BIDMC IRB before implementation.

\section{Inclusion and exclusion criteria}

Patients are deemed eligible for enrollment if they are aged $\geq 18$ years and are scheduled to undergo cardiac surgery with $\mathrm{CPB}$. Patients undergoing off-pump procedures (not requiring $\mathrm{CPB}$ ) are not included, as this study aims to evaluate the role of anesthetic type following subsequent IR lung injury following CPB. Patients are excluded if they are having emergency surgery, have a history of severe chronic obstructive or restrictive lung disease (as evident by spirometry), recent (within two weeks) use of systemic glucocorticoids, prior history of pneumothorax, allergy or contraindication to propofol, or have a personal family history, or are at increased risk of malignant hyperthermia.

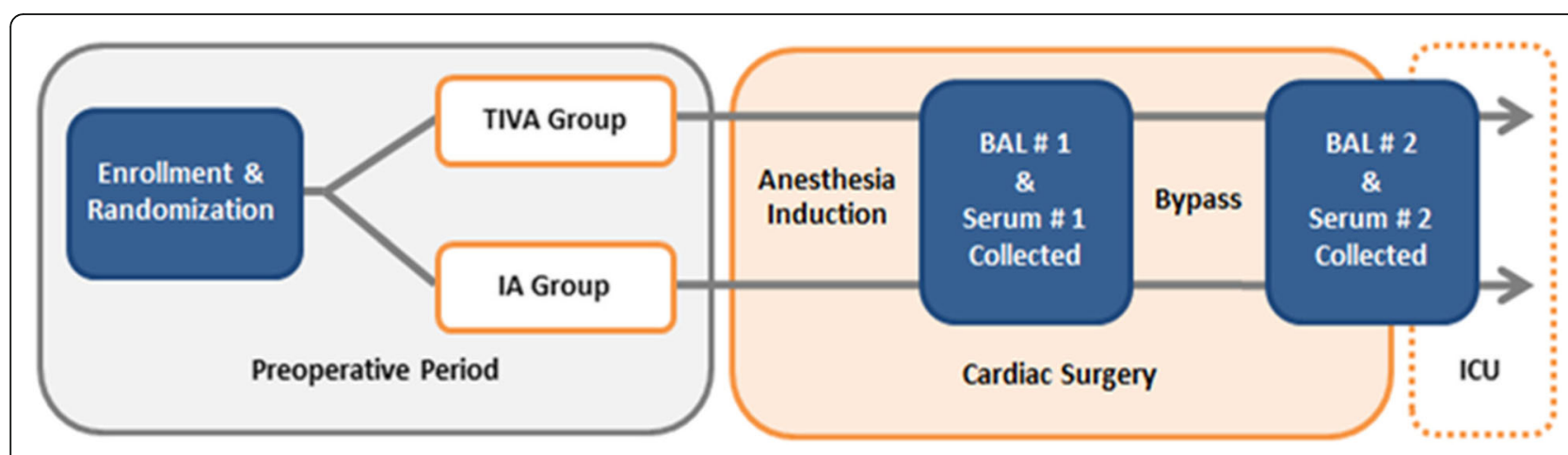

Fig. 1 APLICS study schema. TIVA total intravenous anesthesia (propofol), IA inhaled anesthetic (sevoflurane), ICU intensive care unit 


\begin{tabular}{|c|c|c|c|c|}
\hline \multirow[b]{4}{*}{ TIMEPOINT** } & \multicolumn{4}{|c|}{ STUDY PERIOD } \\
\hline & \multirow{3}{*}{$\begin{array}{c}\begin{array}{c}\text { Enrolment \& } \\
\text { Allocation }\end{array} \\
\text { Prior to } \\
\text { Surgery }\end{array}$} & \multicolumn{3}{|c|}{ Post-allocation } \\
\hline & & \multicolumn{2}{|c|}{ Day of Surgery } & \multirow{2}{*}{$\begin{array}{c}\text { Postoperatively } \\
\text { Daily During } \\
\text { the Hospital } \\
\text { Stay }\end{array}$} \\
\hline & & Pre Bypass & $\begin{array}{l}\text { 2-4 Hours After } \\
\text { Aortic Clamp } \\
\text { Removed }\end{array}$ & \\
\hline \multicolumn{5}{|l|}{ ENROLLMENT: } \\
\hline \multirow{3}{*}{$\begin{array}{l}\text { Eligibility Screen } \\
\text { Informed Consent } \\
\text { Group Allocation }\end{array}$} & $\mathrm{x}$ & & & \\
\hline & $\mathrm{x}$ & & & \\
\hline & $\mathrm{x}$ & & & \\
\hline \multicolumn{5}{|l|}{ INTERVENTIONS } \\
\hline \multirow{2}{*}{$\begin{array}{l}\text { Inhaled Anesthetic (Sevoflurane) } \\
\text { Total Intravenous Anesthesia (Propofol) }\end{array}$} & & $X^{*}$ & & \\
\hline & & $X^{*}$ & & \\
\hline \multicolumn{5}{|l|}{ ASSESSMENTS } \\
\hline Bronchoalveolar Lavage ( $B A L)$ & & $x$ & $\mathrm{x}$ & \\
\hline Blood Collection & & $\mathrm{x}$ & $\mathrm{x}$ & \\
\hline $\begin{array}{l}\text { Assess Intraoperative Protocol } \\
\text { Adherence }\end{array}$ & & $X^{*}$ & & \\
\hline $\begin{array}{l}\text { Assess Postoperative Pulmonary } \\
\text { Complications (PPCs) }\end{array}$ & & & & $\mathrm{x}$ \\
\hline
\end{tabular}

Fig. 2 SPIRIT figure

\section{Randomization}

After informed consent performed by study MDs, participants are allocated in a 1:1 fashion using block randomization to receive intraoperative anesthetic maintenance using either sevoflurane or propofol. The REDCap randomization module hosted at BIDMC is used in order to implement the study randomization schema by study personnel before any study interventions take place [13].

Intervention group: anesthetic maintenance with sevoflurane

Patients in the sevoflurane arm receive anesthetic maintenance (between anesthetic induction and the completion of surgery, including while on $\mathrm{CPB}$ ) with 0.7-1.5 minimum alveolar concentration (MAC) sevoflurane. As clinical conditions may dictate dosing outside of these ranges, for instance in the event of extreme variations in blood pressure, anesthesiologists are asked to maintain an average MAC dose per hour within the specified range.

Comparison group: anesthetic maintenance with propofol Patients randomized to the propofol arm receive anesthetic maintenance with intravenous propofol at a suggested dosing range of $50-200 \mu \mathrm{g} / \mathrm{kg} / \mathrm{min}$. Anesthesiologists are given preference as to the specific dose required to achieve a satisfactory plane of anesthesia and hemodynamic stability, as well as whether to administer additional agents typically included during a total intravenous anesthetic (e.g. remifentanyl) or to use bispectral index monitoring.

Intraoperative clinical management in both groups Anesthetic providers for patients in both groups are asked to avoid the administration of additional potential 
modulators of lung injury including nitrous oxide, steroids, and cisatracurium.

As mechanical ventilation can potentially induce confounding inflammatory lung injury and the administration of a high fraction of inspired oxygen $\left(\mathrm{FiO}_{2}\right)$ can potentially worsen pulmonary IR injury, we have standardized the mechanical ventilation and oxygenation of the study patients according to the following guidelines:

- tidal volume will be set at $6-8 \mathrm{cc} / \mathrm{kg}$ of ideal body weight;

- positive end-expiratory pressure will be set at 2-12 $\mathrm{cmH}_{2} \mathrm{O}$;

- $\mathrm{FiO} 2$ will be $\leq 50 \%$ and titrated to target peripheral oxygen saturation $>92 \%$. Brief exposures $(<20 \mathrm{~min})$ to a $\mathrm{FiO}_{2}$ of $100 \%$ for hypoxia or during the immediate post-CPB period are allowed;

- peak airway pressure goal $<40 \mathrm{cmH}_{2} \mathrm{O}$;

- plateau pressure goal $<35 \mathrm{~cm} \mathrm{H}_{2} \mathrm{O}$;

- arterial partial pressure of carbon dioxide $\left(\mathrm{PaCO}_{2}\right)$ will be maintained at $20-60 \mathrm{mmHg}$;

- arterial partial pressure of oxygen $\left(\mathrm{PaO}_{2}\right)$ will be maintained at $60-200 \mathrm{mmHg}$.

Protocol adherence will be facilitated by real-time conversations between the study team and the anesthesiologist; post hoc quantification of adherence will be performed by review of the anesthetic record.

\section{Drop-out criteria}

The anesthesia team has the discretion to terminate the study protocol at any time in the interest of patient safety. This would include decisions made by the anesthesiologist to deliver a different anesthetic or to administer a steroid, for example in the event of suspected anaphylaxis. In addition, study participants will not be included in the analysis of the primary outcome if the patient is deemed at high risk for complications during a study bronchoscopy and BAL samples cannot be obtained. This includes instances of severe hypoxia (defined as a $\mathrm{PaO}_{2} / \mathrm{FiO}_{2}$ ratio $<100$ or oxygen saturation $\left[\mathrm{SpO}_{2}\right]<90 \%$ ), positive end-expiratory pressure values > $15 \mathrm{cmH}_{2} \mathrm{O}$, or patients with fraction of inspired oxygen $\left(\mathrm{FiO}_{2}\right)>80 \%$ in order to maintain a $\mathrm{SpO}_{2} 90 \%$. Finally, the study intervention can be terminated at any time due to significant nursing or treating physician concern.

\section{Blinding}

This is an unblinded trial. The primary outcome of the study relies on the measurement of laboratory values which are not influenced by provider bias. Furthermore, effective blinding of the anesthesia team or the study group members to randomization assignment would require the use of both a lipid emulsion placebo to mimic the administration of propofol and a sham vaporizer to mimic the administration of sevoflurane. Additionally, study members who assess secondary outcomes are limited to the identification of PPCs via chart review as documented by the treating clinical team, laboratory values, and radiographic data as interpreted by independent radiologists uninformed of study participation or group assignment.

\section{Study procedures: sample collection}

BAL and serum collection take place at two clearly defined time points. The first sample collection occurs after anesthetic induction with endotracheal intubation and before surgical incision. The second sample collection takes place 2-4 h after removal of the aortic cross clamp. This window was chosen as it historically has been described as the time when the peak pulmonary inflammatory response occurs after reperfusion [4]. All BAL samples are obtained by a member of the study team with the appropriate training according to current BIDMC policy and procedure for BAL collection. Direct vision bronchoscopy is utilized for this purpose to minimize the risk of trauma from unintentional misplacement of a catheter. A flexible bronchoscope is passed through the endotracheal tube and gently wedged into the right lower lobe bronchus. A total of $60 \mathrm{cc}$ of a sterile saline solution, typically in 30-cc instillations, is then used to lavage the cavity. Continuous suction is then used to obtain the residual lavage specimen (usually $10-20 \mathrm{cc}$ ) for laboratory analysis of the markers indicated below. Serum collection consists of one $10 \mathrm{cc}$ ethylenediaminetetraacetic acid (EDTA) tube per time point and is obtained via catheters placed as part of the usual care for cardiac surgery.

\section{Specimen processing}

Blood and BAL specimens are centrifuged at $25^{\circ} \mathrm{C}$ at $2300 \times \mathrm{g}$ for $10 \mathrm{~min}$. The plasma and buffy coat are separated from red blood cells, aliquoted into smaller cryovials, labeled and frozen at $-80^{\circ} \mathrm{C}$ for subsequent batch analysis. The BAL specimen is also aliquoted, labeled, and frozen in a similar fashion. All specimens are labeled with a unique coded ID and will not contain any patient identifiers.

\section{Data collection}

Clinical and demographic variables are collected and reported including age, body mass index, Society of Thoracic Surgeons (STS) predicted mortality score, and co-morbidities (e.g. history of myocardial infarction, congestive heart failure, diabetes, dyslipidemia, hypertension, stroke, peripheral vascular disease, and smoking). All patient data are stored anonymously in a REDCap database hosted at BIDMC. REDCap is a 
secure, web-based application designed to support data capture for clinical trials that allows customized datacollection fields to support individual trial needs. Members of the research team are responsible for building and maintaining the electronic case report form, as well as monitoring data entry for completeness, timeliness, and accuracy. During the informed consent process, individuals are asked separately to consent to specimen storage for use in future studies.

\section{Primary outcome: degree of inflammatory lung injury}

The degree of inflammatory lung injury will be assessed by comparing the levels of key inflammatory mediators and biomarkers of lung injury found in patient BAL fluid and serum before and after exposure to cardiopulmonary bypass. Although to date there has not been a clear consensus on the ideal biomarker of pulmonary injury, we have chosen TNF $\alpha$ as our primary outcome to be consistent with the findings of studies done in thoracic surgery where the exposure to pulmonary IR injury is similar to cardiac surgery [8]. In addition to TNF $\alpha$, the indicators tested for as secondary outcomes in this study will likely include interleukin (IL) 1b/6/8, monocyte chemoattractant protein (MCP) 1 , total protein, neutrophil count, soluble receptor for advanced glycosylation end products (sRAGE), angiopoietin 1 and 2 , surfactant protein $\mathrm{D}$, and soluble intercellular adhesion molecule 1 (ICAM1). These indicators have been implicated as markers of lung inflammation and injury in previous studies [14]. Our list of indicators will potentially be expanded as additional novel biomarkers that are currently unknown but available at the time of analysis are identified.

\section{Secondary outcome: incidence of postoperative pulmonary complications}

The incidence of pulmonary complications will be observed between both groups until discharge. PPCs are defined as a composite of: ARDS according to the Berlin Criteria, atelectasis, pleural effusion, pneumonia, pneumothorax, bronchospasm, exacerbation of chronic lung disease, reintubation, or ventilator dependence $>48 \mathrm{~h} \mathrm{[15]}$. This definition is in accordance with the recent consensus definition of PPCs reached by the ESA/ ESICM taskforce and ARISCAT risk scoring system $[16,17]$. In addition, the incidence of hypoxia $\left(\mathrm{PaO}_{2} /\right.$ $\mathrm{FiO}_{2}<300$ ) and respiratory acidosis (partial pressure of carbon dioxide $>45 \mathrm{mmHg}$ ) will be included in a separate composite model, in light of the potential relation of hypoxia and hypercarbia to lung injury and the clinical consequences of these findings with regards to patient management.

\section{Reporting of compliance and adverse events}

A specialist within the research group will monitor protocol compliance, occurrence, and reporting of adverse events to the IRB.

\section{Sample size and power}

A previous trial evaluating the effect of sevoflurane versus propofol during thoracic surgery found a $40 \%$ relative reduction in post-injury increase of alveolar TNF $\alpha$ for the sevoflurane group [8]. Based on this effect size estimate, and assuming a two-sided $\alpha$ of 0.05 and $80 \%$ power, we estimate that 32 participants would be required to detect a difference of $>40 \%$ in alveolar TNF $\alpha$ concentrations between groups. We aim to ultimately enroll a total of 20 individuals per group in order to assess potential differences in other inflammatory markers as secondary outcomes. Additional participants may be enrolled in order to ensure a total of 40 analyzable datasets are obtained after accounting for potential withdrawal of patients before randomization (e.g. surgery changes, patient withdrawal). Analysis of our secondary aims will likely be underpowered but will be used to identify the incidence of PPCs and support future power calculations into the relationship between anesthetic use and PPCs.

\section{Statistical analysis}

We will use SAS software version 9.4 or later (SAS Institute, Cary, NC, USA) to conduct all analyses. Descriptive statistics of the data will be assessed and presented as mean ( \pm standard deviation), median (interquartile range), or frequencies and proportions depending on variable type and distribution. Normality will be assessed with the use of the Shapiro-Wilk test. Differences between groups in continuous variables will be compared using parametric or non-parametric t-tests as appropriate. Categorical data will be compared using a Chi-square or Fisher's exact test for small cell counts. Two-sided $p$ values $<0.05$ will be considered statistically significant for all analyses.

\section{Analysis of the primary outcome}

Our primary outcome, differences in BAL TNF $\alpha$ levels found before and after exposure to $\mathrm{CPB}$, will be assessed using a paired $\mathrm{t}$-test or Wilcoxon signed rank sum test as appropriate. A similar analysis will be performed for additional BAL and serum biomarkers as secondary outcomes. It is anticipated that randomization should account for potential differences between groups at baseline; however, more sophisticated adjustment, for example with multivariable logistic regression, for potential confounders may occur if differences between groups persist after randomization in relevant categories 
such as surgery type and duration of aortic cross clamping.

\section{Analysis of secondary outcomes: postoperative pulmonary complications}

In order to assess the relationship between anesthetic type and PPCs, logistic regression will be used, with data presented as odds ratios and 95\% confidence intervals. In the event that the outcome is more common than expected ( $>10 \%$ incidence), we will employ the use of log-binomial regression, presenting relative risk estimates and their confidence intervals. Individual outcomes will also be reported to see if any early trends emerge between the sevoflurane and propofol groups. In addition, we plan to perform two subgroup analyses of PPCs, one stratified by baseline risk and the other including the variables for hypoxia and hypercarbia. Risk will be calculated according to the ARISCAT preoperative pulmonary risk score and assessed using quartiles in order to reduce the potential for results to be skewed by the distribution of the data. Components of the ARISCAT scores will be calculated for each patient based off their ASA classification, functional status, and presence of preoperative sepsis. Similar regression analyses will then be used to ascertain differences between anesthetic groups and incidence of PPCs in each strata of risk.

\section{Protocol funding sources and their role}

This study is supported by the American Society of Anesthesiologists' Foundation for Anesthesia Education and Research. Funds have been allotted from this organization to support principal investigator time and effort. The scientific content of the study protocol and execution of the trial is in no way influenced by this funding source. Protocol development, execution, and adherence, as well as scientific content development are supported under the Center for Anesthesia Research Excellence (CARE) within the Department of Anesthesia, Critical Care and Pain Medicine at BIDMC.

\section{Discussion}

The APLICS trial will be the first to evaluate the potential for anesthetic choice to reduce inflammatory lung injury after cardiac surgery. Given the high incidence of PPCs in this cohort, a reduction in inflammation could theoretically lead to an improvement in outcomes for an increasingly large group of at-risk patients. Recently, a similar trial conducted in lung cancer surgery patients demonstrated a significant reduction in PPC incidence from the use of sevoflurane compared to propofol [18]. The proposed mechanism for this finding was a decrease in pulmonary inflammation as evident by a reduction in the release of pulmonary pro-inflammatory cytokines in the sevoflurane group. A similar result from our trial would add to the growing body of evidence that the use of the inhaled anesthetics including sevoflurane for surgical anesthesia can lead to a reduction in pulmonary inflammation and potentially improved clinical outcomes.

Our study has several limitations. First, APLICS is not powered to detect a difference in clinical outcomes. The reasons for this design choice are multifactorial. Although the mechanism for lung injury in thoracic and cardiac surgery are similar, a reduction in lung inflammation with the use of the inhaled anesthetics compared to propofol has not yet been demonstrated in this patient group. Therefore we thought it important to power a smaller study to detect a difference in chemical inflammatory mediators before conducting a larger clinical trial aimed at identifying potential differences in clinical outcomes. A second limitation of APLICS is the potential for bias that comes with the lack of assessor blinding. As discussed above, the requirements for the adequate intraoperative blinding of the clinical and study teams to the group assignment were thought to be excessive given the objective nature of the primary outcome. A more relevant source of bias through lack of blinding can occur during the assessment of the secondary outcome, PPCs. Our group has taken several steps to reduce this potential source of bias. First, our definition of PPCs is consistent with international consensus. Second, the determination of PPCs is largely made through the interpretation of laboratory results and radiographic data obtained through the course of usual care and thus interpreted by clinical personnel unaware of the study and group assignment. In the cases where there is potential for a subjective assignment of PPCs, for example pneumonia, study staff are restricted to indentifying pneumonia only from chart review of diagnoses made by clinical staff who were unaffiliated with the trial.

Another limitation of our study is the potential for propofol exposure in the sevoflurane group. Clinicans are permited to use any anesthetic induction agent of their choosing, including propofol. Since we hypothesize that the critical exposure period for the anestheticmediated prevention of IR lung injury is during CPB, this potential for contamination between groups was thought to be minimal in comparison to the safety benefit afforded to the clinical team in achieving a safe anesthetic induction in patients at high risk for hemodynamic compromise. Along the same lines, anesthesia and ICU providers were also allowed freedom of choice in deciding the safest and most effective means of postoperative sedation, including the use of propofol infusions. The scientific benefit of maintaining strict control of the study exposure is outweighed by a need to maximize patient safety.

The APLICS trial will be the first to evaluate whether the use of sevoflurane compared to propofol for anesthetic 
maintenance can reduce the degree of inflammatory lung injury following exposure to CBP. A positive result from APLICS would add to a growing body of evidence supporting the potential lung protective effects of the volatile anesthetics. If such a finding could potentially lead to a reduction in patient morbidity by limiting PPCs, it could mean that the simple intervention of anesthetic choice could impact the successful recovery of thousands of cardiac surgical patients per year.

\section{Additional files}

Additional file 1: SPIRIT checklist. (DOC $123 \mathrm{~kb}$ )

Additional file 2: WHO Trial Registration Data Set - Structured Summary. (DOCX $14 \mathrm{~kb}$ )

\section{Abbreviations}

APLICS: Anesthesia to Prevent Lung Injury in Cardiac Surgery; ARDS: Acute respiratory distress syndrome; ARISCAT: Assess Respiratory Risk in Surgical Patients in Catalonia; BAL: Bronchoalveolar lavage; BIDMC: Beth Israel Deaconess Medical Center; CARE: Center for Anesthesia Research Excellence; cc: Cubic centimeter; CONSORT: Consolidated Standards of Reporting Trials; CPB: Cardiopulmonary bypass; ESA: European Society of Anaesthesiology; ESICM: European Society of Intensive Care Medicine; $\mathrm{FiO}_{2}$ : Fraction of inspired oxygen; $\mathrm{H}_{2} \mathrm{O}$ : Water; ICAM: Intercellular adhesion molecule; ICU: Intensive care unit; IL: Interleukin; IRB: Institutional Review Board; Kg: Kilogram; MAC: Minimum alveolar concentration; MCP: Monocyte chemoattractant protein; $\mathrm{PaCO}_{2}$ : Arterial partial pressure of carbon dioxide; PaO2: Arterial partial pressure of oxygen; PPCs: Postoperative pulmonary complications; REDCap: Research Electronic Data Capture; SPIRIT: Standard Protocol Items: Recommendations for Interventional Trials; $\mathrm{SpO}_{2}$ : Peripheral oxygen saturation; sRAGE: Soluble receptor for advanced glycosylation endproducts; STS: Society of Thoracic Surgeons; TNFa: Tumor necrosis factor alpha

\section{Acknowledgements}

The authors would like to acknowledge the contributions from the staff of the BIDMC Center for Research Excellence who assisted greatly in regulatory compliance, data collection, and assessment of secondary outcomes. In addition, the authors would like to thank the members of the BIDMC Cardiac Anesthesia Division. Without their efforts, this study would not have been possible.

\section{Trial status}

The trial is ongoing and currently recruiting. Recruitment began in May 2016 and is expected to be completed in March 2019. The most recent protocol version is 2016P000306/14 (dated 27 August 2018) and was approved by the BIDMC IRB on 15 October 2018.

\section{Authors' contributions}

All authors have contributed to the conception or design, acquisition, analysis, or interpretation of data or manuscript preparations for this study. $\mathrm{BO}$ is the principal investigator and conceived of the idea for this trial along with the senior investigators BS and DT. BO, SS, AM, VBG, BS, and DT drafted, reviewed, and finalized the study protocol. $B O$ and $A M$ developed the statistical analysis plan. BO, BS, SS, AM, VBG, and DT have been involved in drafting and critically revising the manuscript. All authors have read and approved the final version of this manuscript.

\section{Funding}

This study is supported by the Foundation for Anesthesia Education and Research (Mentored Training Research Grant). Funds have been allotted from this organization to support principal investigator time and effort. Protocol development, execution, and adherence, as well as scientific content development are supported by the Center for Anesthesia Research
Excellence (CARE) within the BIDMC Department of Anesthesia, Critical Care and Pain Medicine.

Dr. O'Gara is funded by a Foundation of Anesthesia Education and Research (FAER) Mentored Research Training Grant. Dr. Talmor is funded by grants from the $\mathrm{NIH} / \mathrm{NHLBI}$ and from the Department of Defense/US Army.

\section{Availability of data and materials}

Data from the current study is available from the corresponding author upon reasonable request.

\section{Ethics approval and consent to participate}

This study has been approved by the Committee on Clinical Investigations at Beth Israel Deaconess Medical Center (IRB Protocol no. 2016P000306). Written informed consent will be obtained from all study participants.

\section{Consent for publication}

Not applicable.

\section{Competing interests}

The authors declare that they have no competing interests.

\section{Publisher's Note}

Springer Nature remains neutral with regard to jurisdictional claims in published maps and institutional affiliations.

Received: 12 November 2018 Accepted: 6 May 2019

Published online: 31 May 2019

\section{References}

1. Canet J, Gallart L, Gomar C, Paluzie G, Valles J, Castillo J, et al. Prediction of postoperative pulmonary complications in a population-based surgical cohort. Anesthesiology. 2010;113(6):1338-50.

2. Cueva LF, Bausili M, González R, Ardila C, Galán J. Postoperative pulmonary complications in cardiac surgery patients: a prospective cohort, singlecentre study: 5AP2-8. Eur J Anaesthesiol. 2014;31:80.

3. Milot J, Perron J, Lacasse Y, Letourneau L, Cartier PC, Maltais F. Incidence and predictors of ARDS after cardiac surgery. Chest. 2001;119(3):884-8.

4. Hall RI, Smith MS, Rocker G. The systemic inflammatory response to cardiopulmonary bypass: pathophysiological, therapeutic, and pharmacological considerations. Anesth Analg. 1997;85(4):766-82.

5. Apostolakis EE, Koletsis EN, Baikoussis NG, Siminelakis SN, Papadopoulos GS. Strategies to prevent intraoperative lung injury during cardiopulmonary bypass. J Cardiothorac Surg. 2010;5:1.

6. Ferrando C, Aguilar G, Piqueras L, Soro M, Moreno J, Belda FJ. Sevoflurane, but not propofol, reduces the lung inflammatory response and improves oxygenation in an acute respiratory distress syndrome model: a randomised laboratory study. Eur J Anaesthesiol. 2013;30(8):455-63.

7. De Conno E, Steurer MP, Wittlinger M, Zalunardo MP, Weder W, Schneiter D, et al. Anesthetic-induced improvement of the inflammatory response to one-lung ventilation. Anesthesiology. 2009;110(6):1316-26.

8. Schilling T, Kozian A, Senturk M, Huth C, Reinhold A, Hedenstierna G, et al. Effects of volatile and intravenous anesthesia on the alveolar and systemic inflammatory response in thoracic surgical patients. Anesthesiology. 2011; 115(1):65-74.

9. Strosing KM, Faller S, Gyllenram V, Engelstaedter H, Buerkle H, Spassov S, et al. Inhaled anesthetics exert different protective properties in a mouse model of ventilator-induced lung injury. Anesth Analg. 2016;123(1):143-51.

10. O'Gara B, Talmor D. Lung protective properties of the volatile anesthetics. Intensive Care Med. 2016:42(9):1487-9.

11. Li F, Yuan Y. Meta-analysis of the cardioprotective effect of sevoflurane versus propofol during cardiac surgery. BMC Anesthesiol. 2015;15:128.

12. Shander A, Fleisher LA, Barie PS, Bigatello LM, Sladen RN, Watson CB. Clinica and economic burden of postoperative pulmonary complications: patient safety summit on definition, risk-reducing interventions, and preventive strategies. Crit Care Med. 2011;39(9):2163-72.

13. Harris PA, Taylor R, Thielke R, Payne J, Gonzalez N, Conde JG. Research electronic data capture (REDCap)--a metadata-driven methodology and workflow process for providing translational research informatics support. J Biomed Inform. 2009;42(2):377-81.

14. Ware LB, Calfee CS. Biomarkers of ARDS: what's new? Intensive Care Med. 2016;42(5):797-9. 
15. Force TADT. Acute respiratory distress syndrome: The berlin definition. JAMA. 2012;307(23):2526-33.

16. Jammer I, Wickboldt N, Sander M, Smith A, Schultz MJ, Pelosi P, et al. Standards for definitions and use of outcome measures for clinical effectiveness research in perioperative medicine: European Perioperative Clinical Outcome (EPCO) definitions: a statement from the ESA-ESICM joint taskforce on perioperative outcome measures. Eur J Anaesthesiol. 2015; 32(2):88-105.

17. Canet J, Gallart L. Predicting postoperative pulmonary complications in the general population. Curr Opin Anaesthesiol. 2013;26(2):107-15.

18. de la Gala F, Pineiro P, Reyes A, Vara E, Olmedilla L, Cruz P, et al. Postoperative pulmonary complications, pulmonary and systemic inflammatory responses after lung resection surgery with prolonged onelung ventilation. Randomized controlled trial comparing intravenous and inhalational anaesthesia. Br J Anaesth. 2017;119(4):655-63.

Ready to submit your research? Choose BMC and benefit from:

- fast, convenient online submission

- thorough peer review by experienced researchers in your field

- rapid publication on acceptance

- support for research data, including large and complex data types

- gold Open Access which fosters wider collaboration and increased citations

- maximum visibility for your research: over $100 \mathrm{M}$ website views per year

At $\mathrm{BMC}$, research is always in progress.

Learn more biomedcentral.com/submissions 